\title{
The IMF bending upstream of the Martian bow shock
}

\author{
Eduard Dubinin ${ }^{1,2,4}$, Konrad Sauer ${ }^{1,4}$, Magda Delva ${ }^{3,4}$, and Aleksandr Skalsky ${ }^{2,4}$ \\ ${ }^{1}$ Max-Planck-Institut für Aeronomie, D-37191 Katlenburg-Lindau, Germany \\ ${ }^{2}$ Space Research Institute, Russian Academy of Sciences, 117810 Moscow, Russia \\ ${ }^{3}$ Space Research Institute, Austrian Academy of Sciences, Inffeldgasse, 12, 8010, Graz, Austria \\ ${ }^{4}$ International Space Science Institute (ISSI), Hallerstrasse 6, 3012 Bern, Switzerland
}

(Received August 4, 1997; Revised January 19, 1998; Accepted February 9, 1998)

\begin{abstract}
A noticeable bending of the magnetic field lines in the Martian upstream region is observed. Variations of the field are controlled by the IMF and the foreshock geometry. The sign of $\Delta \boldsymbol{B}$ changes at the crossing of the tangent surface, which borders foreshock and the undisturbed solar wind. Perturbations of the magnetic field are centered at the plane which is normal to the tangent surface and intersects the bow shock at the tangent point. All observed signatures suggest that the current, providing field variations, is localized near the tangent surface. Although, two models are discussed, neither of them is able to explain all observed features satisfactorily.
\end{abstract}

\section{Introduction}

Characteristic features of the Martian bow shock may become very different from those of the Earth's bow shock reason why the former is much smaller than the latter. At Mars, the upstream ion gyroradius is comparable with the curvature radius of the shock and ions reflected from the shock can travel significant distances along the shock surface and smear a difference between a quasi-perpendicular and a quasi-parallel shock (Moses et al., 1989). Another important feature of the Martian environment is the existence of an extended neutral exosphere far beyond the bow shock. Due to the presence of pickup ions and their kinetic effects the situation at Mars upstream may be different from that of Earth or Venus. Picked-up exospheric protons, reflected from the electrostatic barrier at the bow shock, may supply "overreflection" of protons and contribute significantly to the population of backstreaming ions (Dubinin et al., 1994, 1995). Martian ions can generate the wave activity not only in the foreshock region, what is happening in the earth, but also upstream of the tangent line (Delva and Dubinin, 1998; Skalsky et al., 1998). As a consequence, extension of the foreshock study to include the Martian case would be beneficial. In this paper discussion is focused on the study of large-scale variations of the magnetic field. Strong perturbations of the IMF near the border of the Martian foreshock were observed by Dubinin et al. (1995). It was thought that perturbations occur due to distortion of the IMF by ion currents generated by bunch of reflected planetary protons. Below we analyze this feature of the Martian upstream in more detail.

\section{Observations}

To address the question about large scale variations of the magnetic field in the upstream region, a study was carried

Copy right (C) The Society of Geomagnetism and Earth, Planetary and Space Sciences (SGEPSS); The Seismological Society of Japan; The Volcanological Society of Japan; The Geodetic Society of Japan; The Japanese Society for Planetary Sciences. out on data from the MAGMA magnetometer onboard the Phobos-2 spacecraft. After the Phobos-2 was transferred into a circular orbit with $R=2.8 R_{\mathrm{M}}$, which was close to the ecliptic plane, the orbit was very appropriate for the study of the Martian environment, providing a good coverage of the upstream/foreshock interface. Figure 1(a) shows projections of the magnetic field vectors onto the $X Y$-plane in the MarsSun Orbital Reference (MSOR) frame ( $X$-axis to Sun, $Y$-axis opposite to the planet orbital velocity) in the upstream region of the bow shock for four orbits when the IMF had the outward sector polarity. The bow shock was usually crossed near the terminator and was not seen in this display in most cases. Magnetic field was measured every 45 seconds, but vectors are shown with $3.75 \mathrm{~min}$ resolution in Fig. 1. Distinct short scale perturbations, marked by asterisks, are imposed on large scale turn of the IMF. Figure 1(b) presents the data for four other circular orbits when the $Y$-component of the IMF was negative. Similar signatures of the field rotations along the orbit are observed. Variations of the field have common features that suggests their origin in the solar wind/Mars interaction. In order to analyze a large-scale field structure, the $\boldsymbol{V} \boldsymbol{B}$ reference frame was applied to define a coordinate system $\left(X^{\mathrm{m}}, Y^{\mathrm{m}}, Z^{\mathrm{m}}\right)$, where the $X^{\mathrm{m}}$-axis points to the Sun, the $Y^{\mathrm{m}}$-axis is taken in the direction of the component of $\boldsymbol{B}$ perpendicular to the solar wind speed (and thus always $B_{y} \geq 0$ ), the $Z^{\mathrm{m}}$-axis completes the right-hand system. To identify the position of the spacecraft relative to the bow shock, the three dimensional bow shock surface was approximated by a half ellipsoid of revolution around the $X^{\mathrm{m}}$ axis. In the magnetic equatorial plane, the equation of the ellipse with focus at the planet's center is given by $r=\left[r_{\mathrm{ss}}(1\right.$ $+e) /(1+e \cdot \cos \varphi)]$, where $r_{\mathrm{ss}}$ is the bow shock subsolar distance, $e$ is the eccentricity. Observations are reasonably fitted by $r_{\mathrm{ss}}=1.65 R_{\mathrm{M}}$, and $e=0.65$ (Schwingenschuh $e$ t al., 1990; Trotignon et al., 1991). In each plane $Z^{\mathrm{m}}=$ const, the foreshock is determined by the plane's intersection with the bow shock surface and the magnetic field line tangent to this intersection. An angle $\alpha$ between the magnetic field line 
(a)

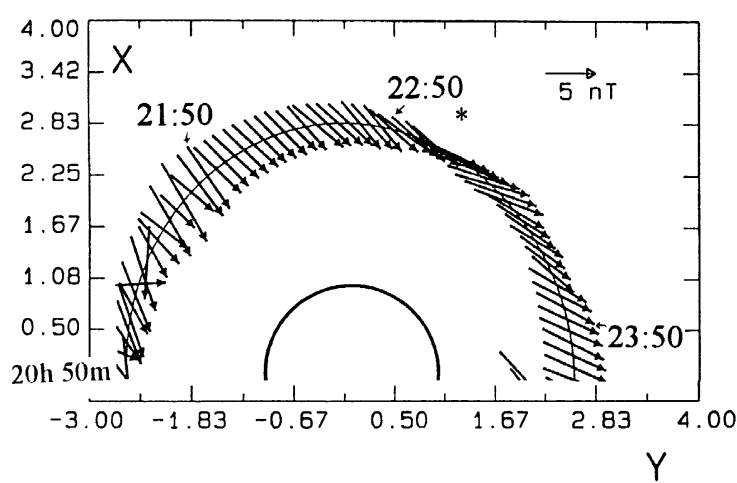

14-MAR-89

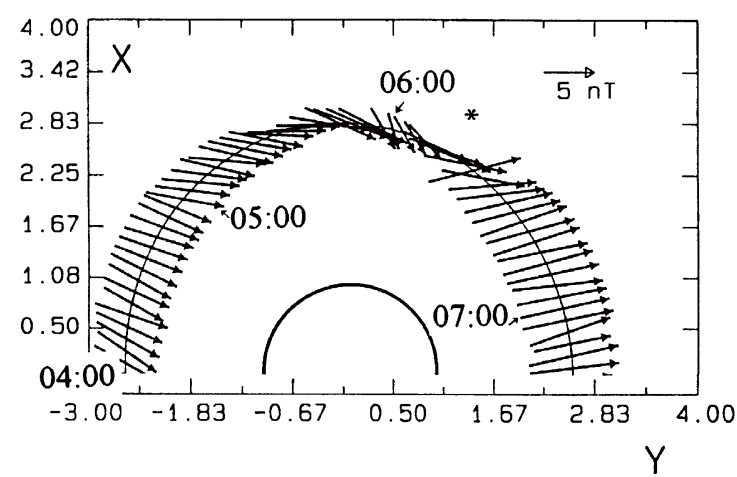

(b)

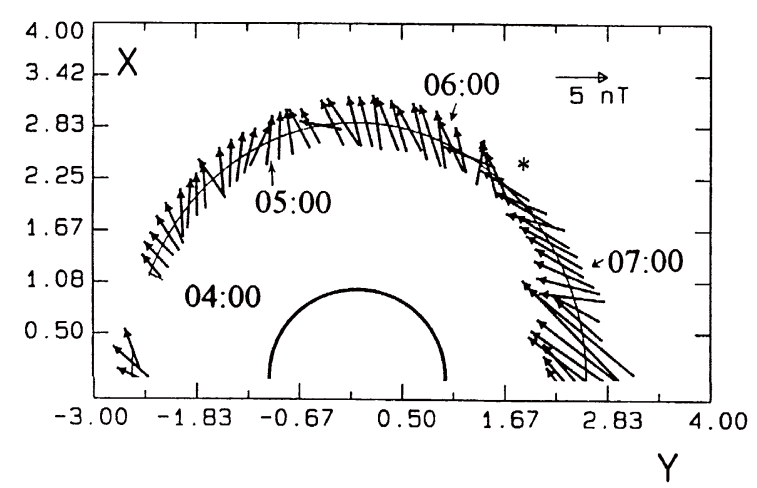

02-MAR-89

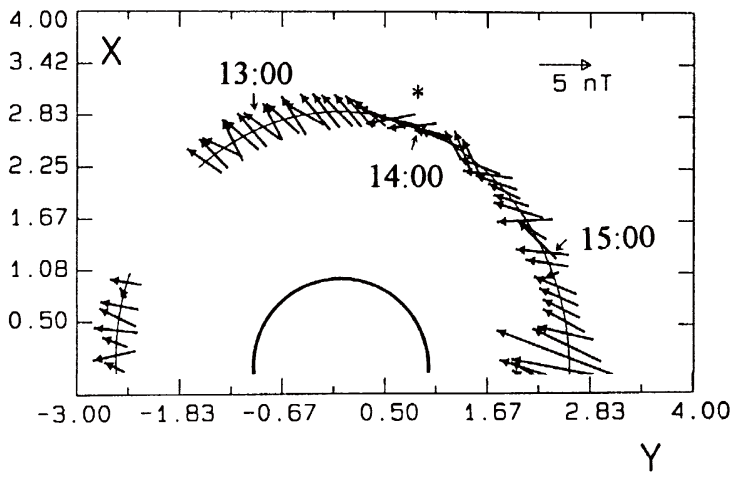

13-MAR-89

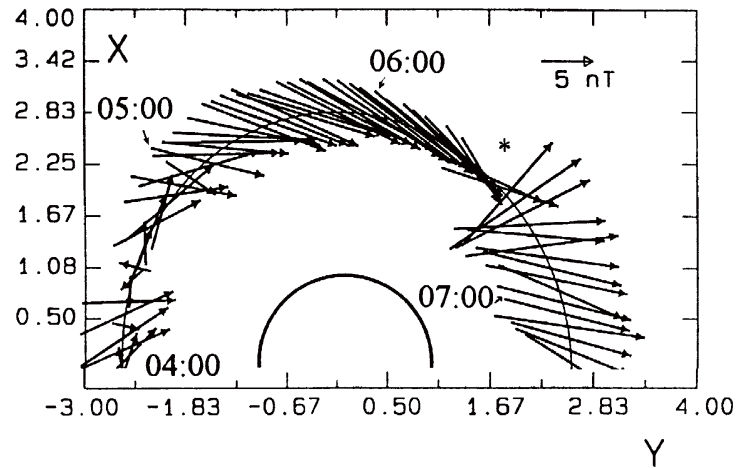

17-MAR-89
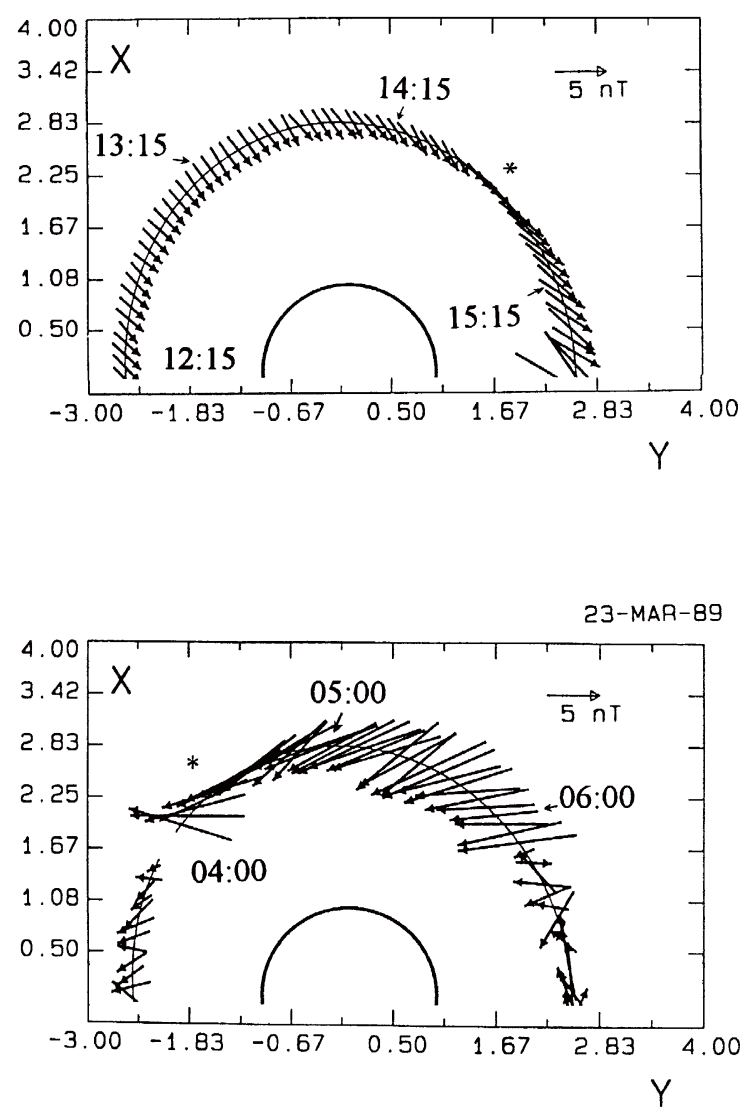

25-MAR-89

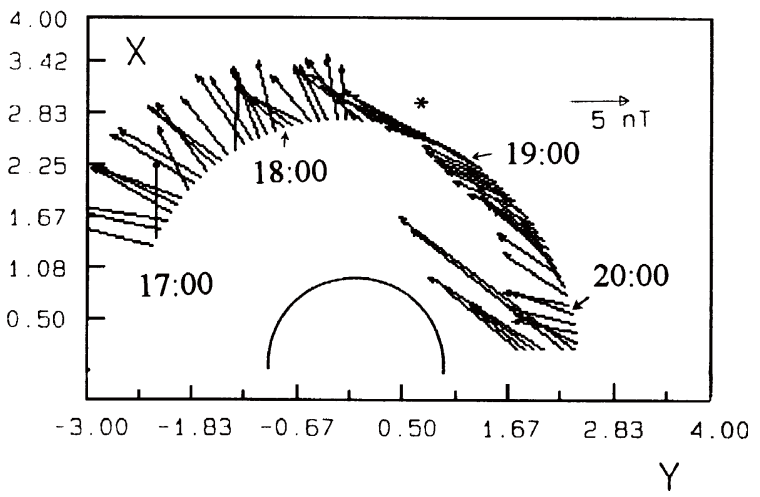

Fig. 1. (a) Projections of the magnetic field vectors onto $X Y$-plane (the Mars-Sun Orbital reference frame) in the upstream region of the bow shock for four orbits with outward IMF sector polarity $\left(B_{y}>0\right)$. (b) The same for four other circular orbits, where the $Y$-component of the IMF was negative. 
tangent to the bow shock and the direction to the spacecraft from the tangent point was evaluated. Positive values of this angle $\left(0^{\circ} \div 180^{\circ}\right)$ correspond to time intervals when the spacecraft was downstream of the tangent line (foreshock region). $\alpha \subset\left(-180^{\circ} \div 0^{\circ}\right)$ gives positions of the spacecraft in the solar wind.

Figures 2(a) and 2(b) show the $B_{x m}$ and $B_{y m}$ components (in the $\boldsymbol{V} \boldsymbol{B}$ frame) and the angle $\alpha$ for several orbits. Left and right vertical dash-dotted lines border the region which is outside of the foreshock $(\alpha<0)$. As far as $B_{x m}$ curves are concerned, tilted dotted lines connect points for $\alpha=0^{\circ}, \pm 180^{\circ}$. Lines for $B_{y m}$ were drawn so that the changes in $\Delta B_{y m}$ becomes similar to those in $\Delta B_{x m}$. A gradual turn of the IMF to be more perpendicular to the solar wind flow, is observed. Although some uncertainty remains in drawing, tilted dotted lines show this trend. Distinct rotations of the magnetic field with respect to this background are also seen when the spacecraft leaves the foreshock and enters the unperturbed solar wind. The $B_{x m}$-component decreases and the transverse component of the field $B_{y m}$ increases. Then, the sign of the disturbance $\Delta B_{x m}$ changes at $\alpha \sim-90^{\circ}$ (middle dash-dotted line). The $\Delta \boldsymbol{B}$ also changes sign across the tangent to the bow shock. The fourth and fifth panels show plasma emissions recorded by the PWS instrument in two frequency ranges. Electron plasma oscillations, generated by suprathermal electrons, that had been accelerated and reflected at the bow shock, is a good indicator of tangent line crossings. A general tendency that plasma emissions appear in the foreshock region, as indicated by $\alpha$-angle, confirms the identification of the upstream region based on the model bow shock and the magnetic field measurements. The fifth panel presents wave emissions measured at frequency of $\sim 100 \mathrm{~Hz}$. Skalsky et al. (1998) indicated that these waves are observed "upstream" of electron plasma oscillations, which have onset near the tangent line, in $30-40 \%$ of all circular orbits. No obvious correlation between waves and the magnetic field bending is found. $100 \mathrm{~Hz}$ waves sometimes accompany a bending of the IMF were observed on March 13 and 25, but not on March 2.

\section{Current Systems}

At a glance, bi-polar variations of the field may be taken to be draping features around the extended Martian exosphere. Indeed, a pickup current of ions originating from the hot oxygen corona could provide a bending of the IMF. However, this simple model meets several serious problems. Figure 3 shows variations of the $B_{x m}$ and $\alpha$-angle for the orbit on March 13, 1989. The trajectory was almost "polar" in the $\boldsymbol{V B}$ coordinates and the spacecraft was traveling near the tangent surface in the foreshock, making only short excursions to the solar wind. The feature of the magnetic field variations, which is inconsistent with a draping pattern, is a change of sign of $\Delta B_{x m}$ across the tangent line. A second inconsistency is that bi-polar variations of the magnetic field are centered at $\alpha \approx-90^{\circ}$ rather than near the subsolar point, in spite of expectation for draping due to the solar wind interaction with the Martian exosphere. Thirdly, a "reversed draping" has been also observed. Figure 4 gives an example of the reversed bending on March 12, 1989. As in the previous cases, a distinct rotation of the magnetic field occurs when the spacecraft crosses the tangent line, but perturbations have an opposite sign.

Dubinin et al.(1995) have supposed that a sudden rotation of the magnetic field across the foreshock boundary might be associated with a current of reflected pickup protons. Using a simple model based on test particle motion in the prescribed electric and magnetic fields with electrostatic barrier at the bow shock, Dubinin et al. $(1994,1995)$ have shown that pickup protons, reflected at the BS, can essentially contribute to the population of backstreaming ions in the foreshock. This model was upgraded in this paper with consideration on probable variations of the IMF due to the current of reflected planetary protons. The current number density $n e V_{\text {ref.pick }}$ was evaluated by tracing trajectories of pickup ions specularly reflected from the electrostatic barrier. It was found that ion current provides perturbations of the magnetic field mainly in the foreshock, and sign of $\Delta \boldsymbol{B}$ is opposite to that was determined from observations. Figure 5 (upper panel) gives variations of the $B_{x}$ component along the spacecraft trajectory (February 8,1989 ) when Phobos2 was in the foreshock with only short touches of the tangent line (lower panel). The solid line shows results of the simulations, which were made with the assumption that the unperturbed IMF was $(1,-2,1)$ which corresponds to the observations made at 04:00 UT. Subsequent variations of the $B_{x}$ are caused by currents of reflected pickup ions. Measured and modeled values are varying in anti phase that indicates a dominance of electron currents. Next example also demonstrates a possible importance of the electron currents. Figure 6 compares observations and a model for the orbit on March 12, 1989 when the spacecraft was mostly upstream of the foreshock. Solid lines show components $\boldsymbol{B}_{\mathrm{o}}$ $+(-\delta \boldsymbol{B})$, where $\delta \boldsymbol{B}$ is the field of ion currents. It is seen that the modeled perturbation, taken with the minus sign, reproduces the trend of field variations. It is worth to be noted that the model does not bring a fine bi(three)-polar structure of disturbances that is clearly observed.

Electron currents can be carried by suprathermal electrons accelerated and reflected at the bow shock. At the Earth's bow shock enhanced fluxes of backstreaming electrons is a main signature of electron foreshock (Feldman et al., 1982). Backstreaming electrons with energies between 100 and $530 \mathrm{eV}$ were also recorded in the Martian electron foreshock (Skalsky et al., 1993). The scheme is shown in Fig. 7, illustrating a map of the magnetic field perturbations $\Delta \boldsymbol{B}$ due to electron currents on the tangent to the bow shock surface. The surface consists of a family of tangent field lines at different $Z_{x m}$ planes. The position of tangent point separates electrons flowing in opposite directions. The sign of $\Delta \boldsymbol{B}$ varies whether the spacecraft is upstream or downstream of the position of the tangent point, or whether the spacecraft is above or below the magnetic equatorial plane. In order to test the model, calculation was made on the spacecraft position in a reference frame $\left(X^{*}, Y^{*}, Z^{*}\right)$ with origin at the tangent point in the magnetic equatorial plane $\left(X^{*}\right.$-axis is along the normal to the tangent surface, $Y^{*}$-axis coincides with the tangent magnetic field line, $Z^{*}$-axis completes the right-hand system). The tangential surface was determined with an assumption of the prescribed IMF in the solar wind. Figure 8 shows the spacecraft location in this reference 

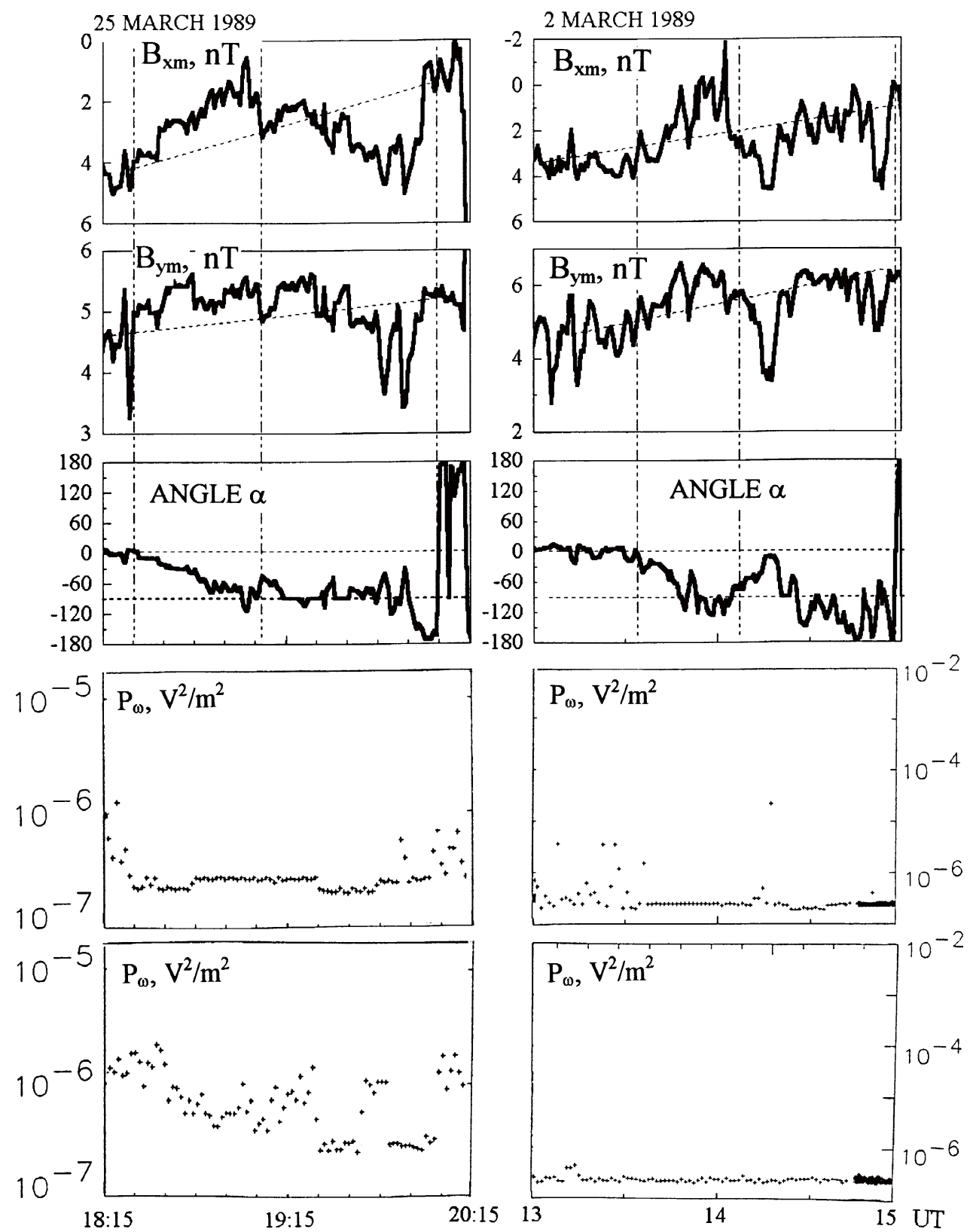

(a)

Fig. 2. $B_{x m}$ and $B_{y m}$ components in the $\boldsymbol{V} \boldsymbol{B}$ reference frame and the angle $\alpha$ between the tangent line and the direction to the spacecraft for several orbits. Left and right vertical dash-dotted lines border the region outside the foreshock $(\alpha<0)$. Two lower panels are intensities of wave emissions $\left(\mathrm{V}^{2} /\right.$ $\mathrm{m}^{2}$ ) at frequencies $6-38 \mathrm{kHz}$ (electron plasma oscillations), and 50-200 $\mathrm{Hz}$.

frame and the perturbations of the IMF for two orbits with "normal" and "reversed" draping signatures. Bottom panels give the angle $\alpha \cdot X^{*}=0$ means that the spacecraft crosses the tangent surface. Signs $-/+$ of the $Y^{*}$-component indicate that the spacecraft occurs upstream or downstream with respect to the position of the tangent point. Crossings of the $Y^{*}\left(X^{*}\right.$ $=0)$ and $X^{*}\left(Y^{*}=0\right)$-axes correspond to $\alpha=-0, \pm 180^{\circ}$ and $\alpha=-90^{\circ}$, respectively. Rotations of the magnetic field are controlled by the spacecraft position with respect to the tangent surface, i.e., by the crossings of the surface itself $\left(X^{*}\right.$ $=0)$, the equatorial plane $\left(Z^{*}=0\right)$, or the plane, which is normal to the tangent surface $\left(Y^{*}=0\right)$. It should be mentioned that sites of critical points are sensitive to the choice of the undisturbed prescribed IMF. The values $B_{x m}=-4,-2$ $\mathrm{nT}, B_{y m}=5.4,6 \mathrm{nT}$ were adopted for orbits on March 12 and 14 respectively. The difference between these two trajectories is that the spacecraft was traveling from the southern hemisphere to the northern hemisphere on March 12, and in the opposite $Z^{*}$ direction on March 14. According to the scheme in Fig. 7, the sign of the field rotation is different from each other. In accordance with the model, perturbations of the IMF must have a three-polar rather than a bi-polar structure along the spacecraft orbit. It is not adequate to note that orbits on March-2, 13, 14, 25 (Fig. 2) give the examples of bi-polar perturbations. A three polar structure is observed in the orbit on March-12. Another important feature is a 

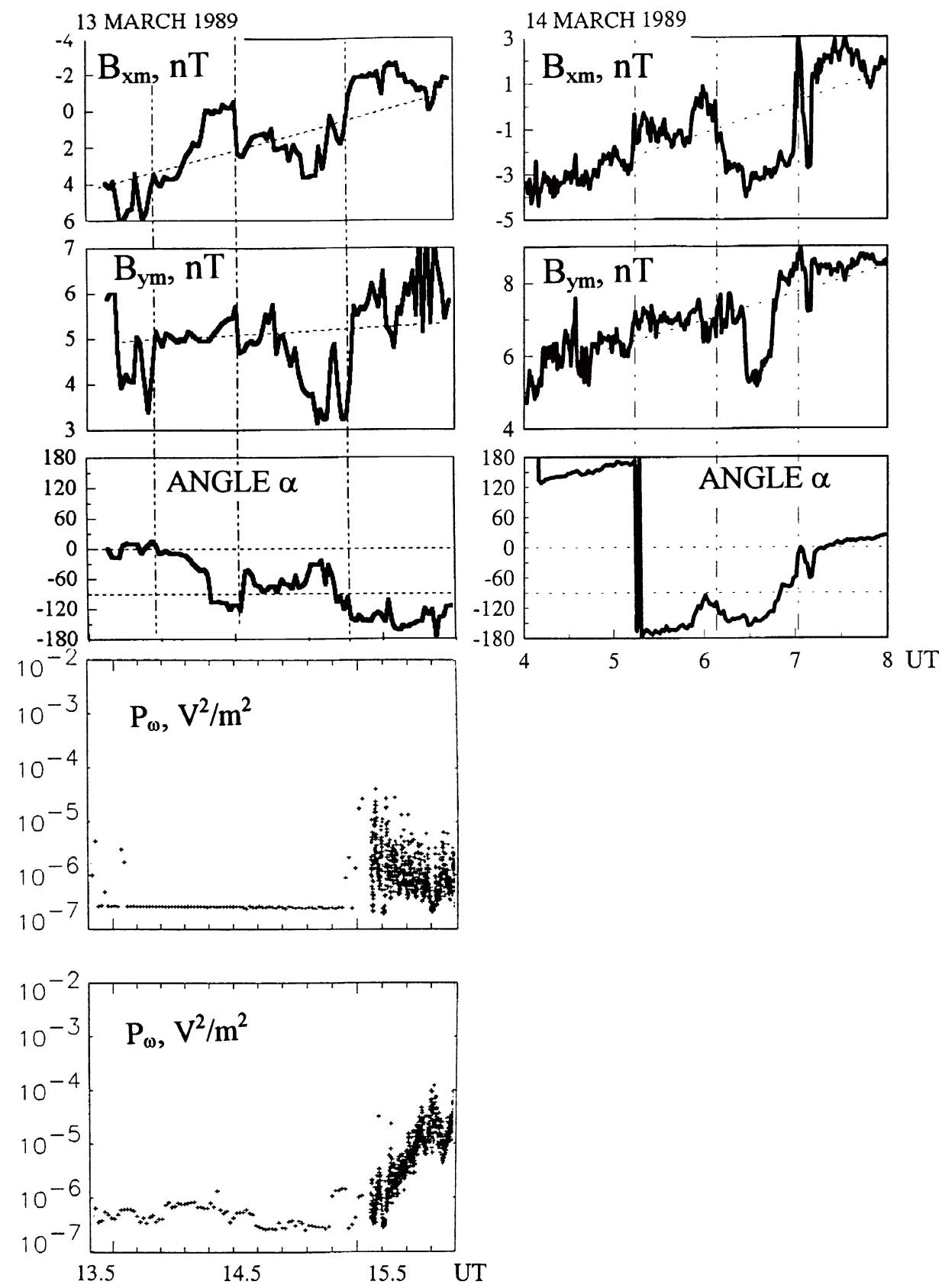

(b)

Fig. 2. (continued)

difference between the methods of tangent line estimation in the model and in the preceding data analysis. "An optimum" IMF direction in the unperturbed solar wind was adopted to calculate angle $\alpha$ and $X^{*}, Y^{*}$ and $Z^{*}$-coordinates of the spacecraft in the model. On the other hand, analyzing the data set, angle $\alpha$ was estimated from each observation point. Both methods can lead to a difference in time of crossing of the tangent surface. For example, according to Fig. 2(b), the spacecraft entered the foreshock region at 07:15 UT (March14), whereas, the model (Fig. 8) brings that the crossing of the tangent line occurs at 08:00 UT. Therefore, independent and reliable identification of the foreshock position becomes important. In some cases we have a good indicator of this crossing from the plasma wave observations. Unfortunately, as was already mentioned, wave measurements of the electron plasma oscillations were not carried out at all orbits.

Addressing the question about the number density of current carriers, we evaluated $n_{\text {cur }} \sim c \cdot \Delta B / 4 \pi L e V_{\text {cur. }}$. Adopting $\Delta B \sim 3 \mathrm{nT}, L \sim 3.10^{8} \mathrm{~cm}, V_{\text {cur }} \sim 5.10^{8} \mathrm{~cm} / \mathrm{s}$ (electrons with energy of about $150-200 \mathrm{eV}$ ), we obtain $n_{\text {cur }}$ $\sim 10^{-3} \mathrm{~cm}^{-3}$. Feldman et al. (1982) have estimated that approximately $10^{-4}$ incident solar wind electrons are accelerated by the Earth's bow shock back into the upstream region, with energy greater than $1 \mathrm{keV}$. Therefore, the number density of about $10^{-3} \mathrm{~cm}^{-3}$ of suprathermal electons $(E \geq 100$ $\mathrm{eV}$ ) carrying current along the tangent surface seems to be a realistic value. However, this model has serious problems because surface electron currents do not provide a normal 
13 MARCH 1989
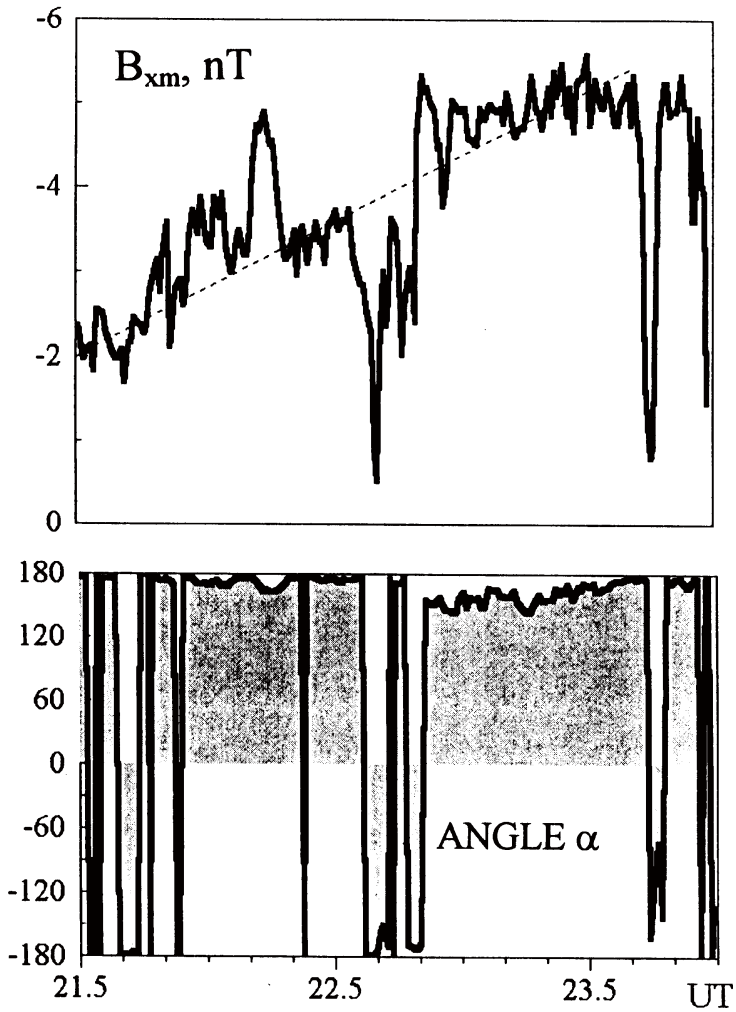

Fig. 3. Variations of the $B_{x m}$ component and $\alpha$-angle (in $\boldsymbol{V B}$ coordinates) for the polar orbit on March 13, 1989.

12 MARCH 1989
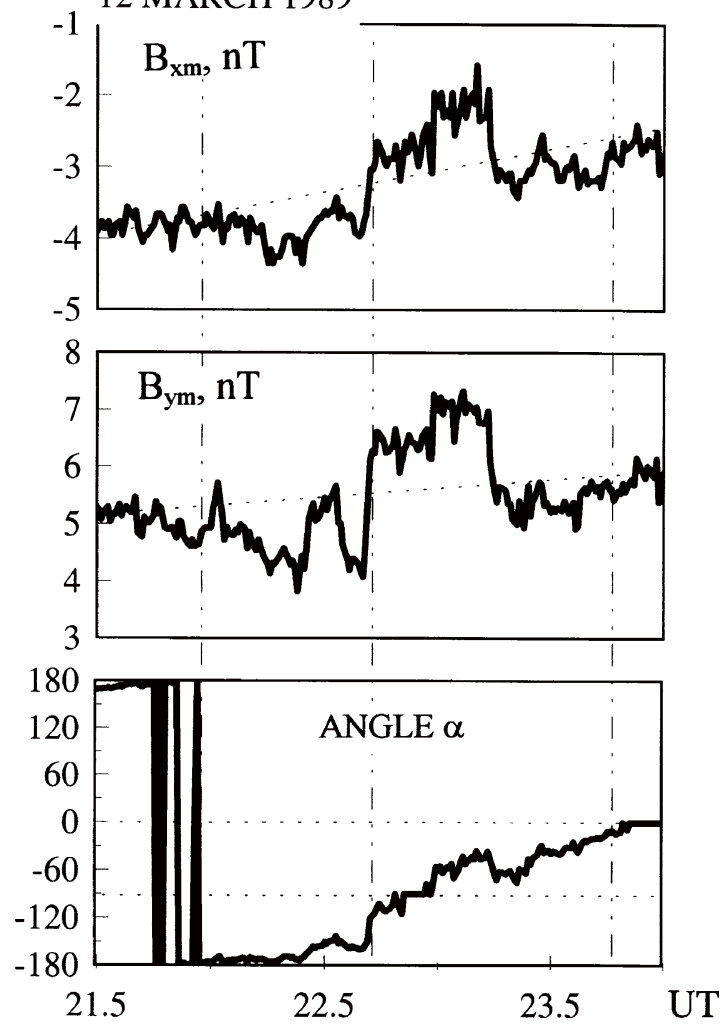

Fig. 4. Example of reversed draping signatures on March 12, 1989.
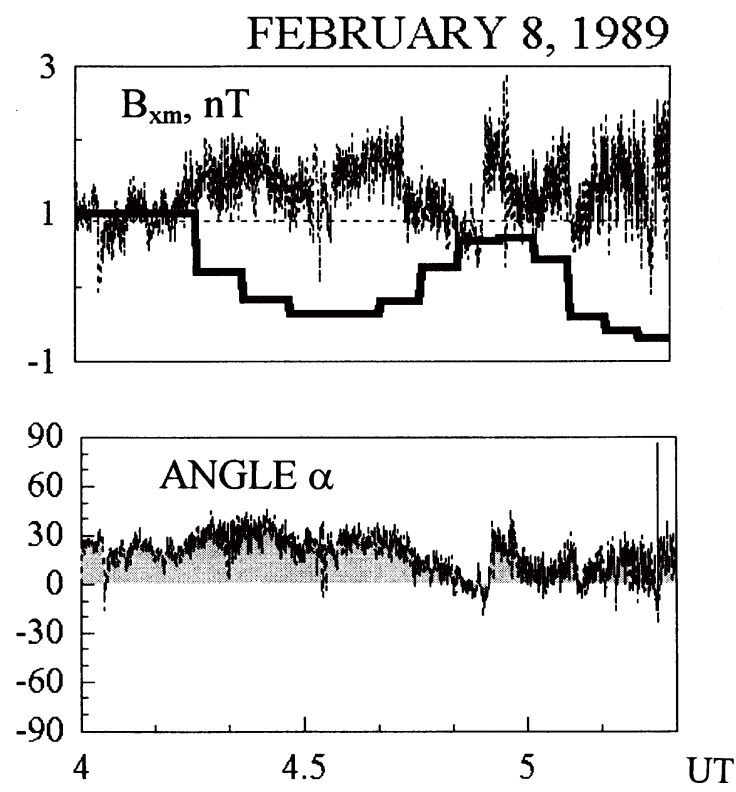

Fig. 5. Variations of the $B_{x}$-component when Phobos-2 was inside the Martian foreshock (February 8, 1989). Solid line gives the modeled perturbed magnetic field caused by currents of pickup exospheric protons, reflected at the bow shock. The bottom panel shows the $\alpha$-angle. Antiphase variations suggest that electron currents may be responsible for magnetic field bending.

field component (bent field lines remain at surfaces parallel to the tangent one). To overcome the problem with the normal component, one may assume that the field lines, which construct a tangent surface, are not straight, giving rise to convexities and concavities of this surface. This prompts a question whether magnetic field lines are strongly sheared near an idealized tangent surface or not.

\section{Summary}

Strong perturbations of the magnetic field are observed, when the Phobos-2 spacecraft left the foreshock region and entered the undisturbed solar wind. These variations comprise a distinct large-scale bending of the IMF and are controlled by the IMF-direction and the foreshock geometry. Disturbances of the magnetic field are centered at the plane which is normal to the tangent surface and intersects the bow shock at tangent points. A change of sign of the $\Delta \boldsymbol{B}$ across the tangent line is also observed. All these signatures suggest that a current, responsible for the field perturbations, is localized near the tangent surface. This surface, formed by a family of field lines tangent to the bow shock at different $Z$-planes, has probably a more complicated shape. Neither of the discussed models gives a satisfactory explanation for the observed features. Since an uncertainty remains always, whether the variations of the magnetic field are associated with changes in the solar wind or if they are internally driven by processes near the Martian foreshock. Only measurements with two spacecrafts would be able to solve this uncertainty. 

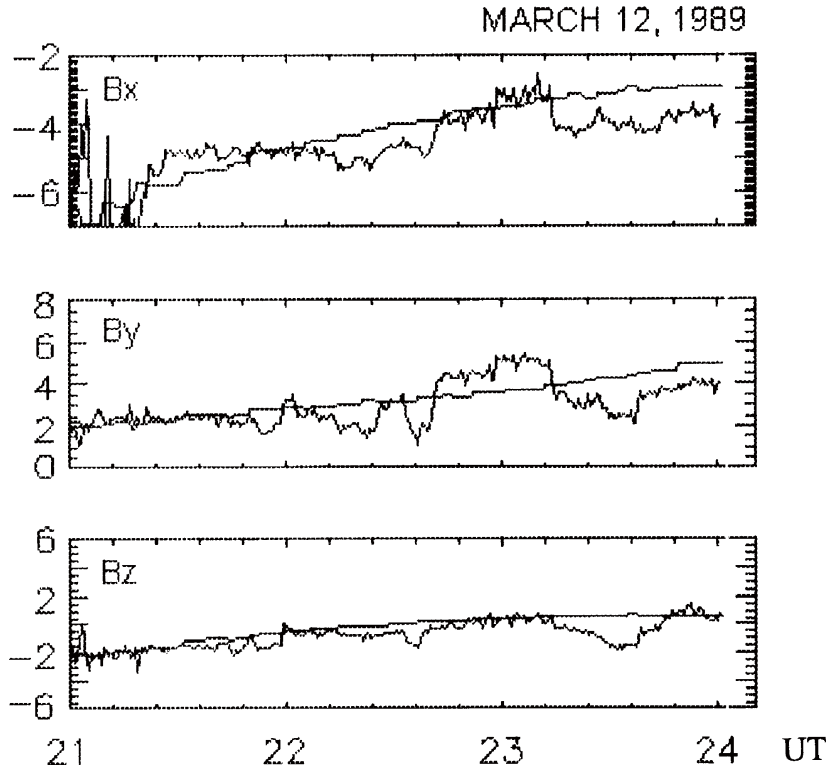

Fig. 6. Comparison of the observed and modeled field variations (the MSOR frame) for the orbit on March 12, 1989.

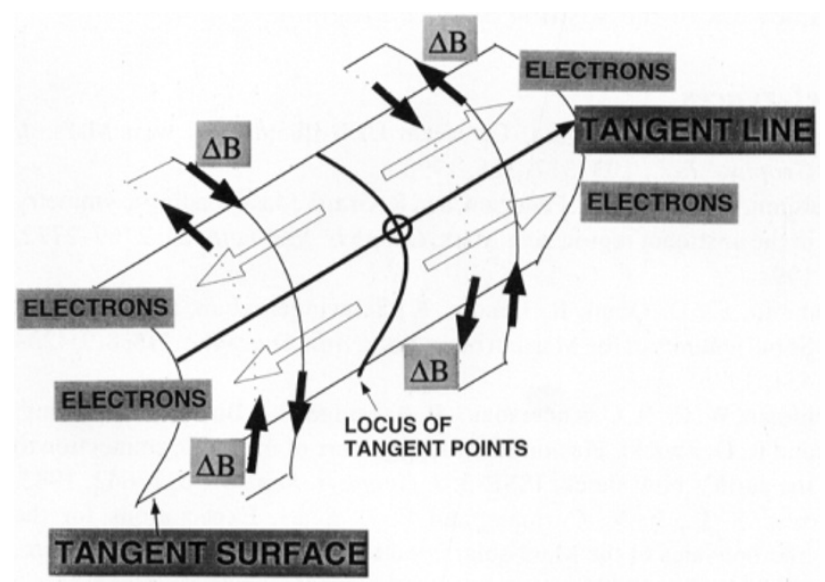

Fig. 7. The scheme of magnetic field perturbations $\Delta \boldsymbol{B}$ due to electron currents at the tangent to the bow shock surface. The sign of $\Delta \boldsymbol{B}$ varies whether the spacecraft is upstream or downstream of the position of the tangent point, or if it is above or below the magnetic equatorial plane.

\section{NORMAL DRAPING}

14 MARCH 1989
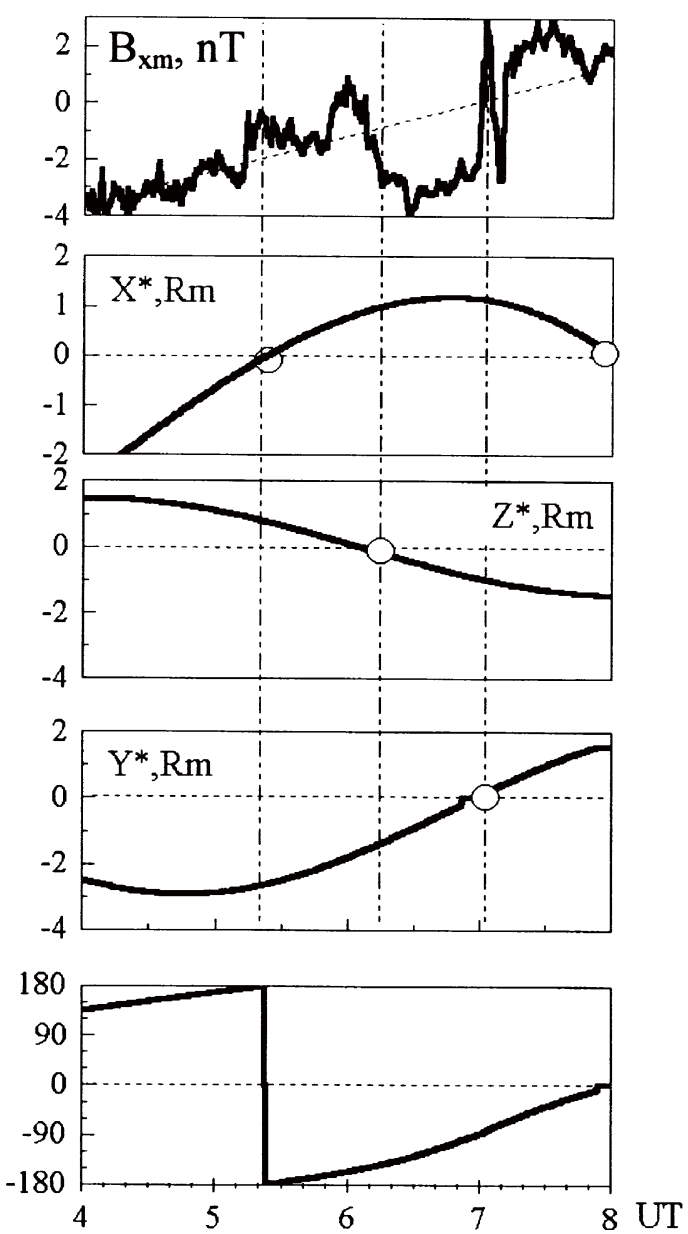

\section{REVERSED DRAPING}

12 MARCH 1989
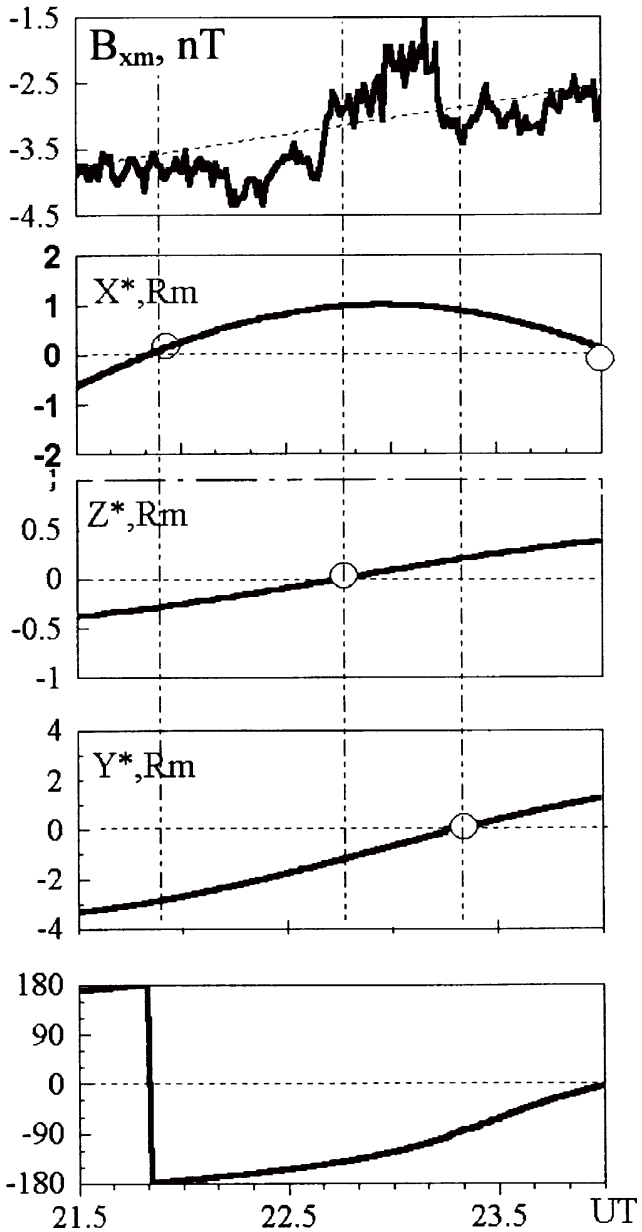

Fig. 8. Magnetic field and coordinates of the spacecraft for two orbits with a "normal" and "reversed" draping signature in the reference frame determined by the foreshock geometry with origin in the central tangent point. 
Acknowledgments. The authors wish to express their thanks to the ISSI in Bern for the support in the collaboration in the framework of the Visiting Science Program.

\section{References}

Delva, M. and E. Dubinin, Upstream ULF fluctuations near Mars, $J$. Geophys. Res., 103, 317-326, 1998.

Dubinin, E., D. Obod, A. Pedersen, and R. Grard, Mass-loading asymmetry in the upstream region near Mars, Geophys. Res. Lett., 21, 2769-2772, 1994.

Dubinin, E., D. Obod, R. Lundin, K. Schwingenschuh, and R. Grard, Some features of the Martian bow shock, Adv. Space Res., 15, 8(9)423431, 1995.

Feldman, W. C., R. C. Anderson, J. R. Asbridge, S. J. Bame, J. T. Gosling, and R. D. Zwickl, Plasma electron signature of magnetic connection to the earth's bow shock: ISSE-3, J. Geophys. Res., 87, 632-642, 1982.

Moses, S. L., F. V. Coroniti, and F. L. Scarf, Expectations for the microphysics of the Mars-solar wind interaction, Geophys. Res. Lett., 15, 429-432, 1989.
Schwingenschuh, K., W. Riedler, H. Lichtenegger, Y. Yeroshenko, K. Sauer, J. G. Luhmann, M. Ong, and C. T. Russell, Martian bow shock: Phobos observations, Geophys. Res. Lett., 17, 889-892, 1990.

Skalsky, A., R. Grard, P. Kiraly, S. Klimov, V. Kopanyi, K. Schwingenschuh, and J. G. Trotignon, Simultaneous plasma waves and electron flux observations upstream of the Martian bow shock, Planet. Space Sci., 41, (3)183-188, 1993.

Skalsky, A., E. Dubinin, M. Delva, R. Grard, S. Klimov, K. Sauer, and J.G. Trotignon, Wave observations at the foreshock boundary in the near-Mars space, Earth Planets Space, 50, 1998 (in press).

Trotignon, J.-G., R. Grard, and S. Klimov, Location of the Martian bow shock: measurements by the plasma wave system on Phobos-2, Geophys. Res. Lett., 18, 365-368, 1991.

E. Dubinin (e-mail: dubinin@helene.mpae.gwdg.de), K. Sauer (e-mail: sauer@linax1.mpae.gwdg.de), M. Delva (e-mail: delva@fiwf01.tu-graz.ac.at), and A. Skalsky (e-mail: askalsky@vm1.iki.rssi.ru) 